\title{
Diagnosis of Hand Tumors using fNIR Optical System
}

\author{
Ebraheem Sultan ${ }^{1}$, Nizar Ahmad ${ }^{1}$ and Afshin S. Daryoush ${ }^{2}$ \\ ${ }^{1}$ Electronic Engineering Department, College of Tech. Studies-PAAET, \\ Kuwait, Kuwait \\ ${ }^{2}$ Department of Electrical and Computer Engineering, Drexel University, \\ Philadelphia, PA, USA
}

\begin{abstract}
The free-space broadband functional near-infrared (fNIR) spectroscopy technique has been used to clinically measure the optical properties of ganglion cysts and lipoma tumors. The NIR system measures the broadband (30MHz-1000MHz) insertion loss (IL) and the insertion phase (IP) of the modulated light. This helps to characterize and model the different penetration depth and path-related modulated photon movement to perform spectroscopic imaging of six patients with right-hand wrist tumors (both ganglion and lipomas). The study is divided into two stages. The first stage is dedicated to performing IL and IP measurements over $30 \mathrm{MHz}-1000 \mathrm{MHz}$ in back-scattering, while in the second stage of broadband measurements using the forward scattering is to extract the ganglion cyst optical absorption and scattering parameters to confirm the diagnosis. The result of the second stage is then used to perform 3D numerical simulation using multi-physics tool (COMSOL).
\end{abstract}

Keywords: fNIR Spectroscopy, Ganglion Cysts, Lipomas Fatty Tumor, optical transmitter, VCSEL, Optical Receiver, APD, IP and IL

\section{Introduction}

Functional medical imaging based on NIR optical spectrum is considered an efficient method for diagnosing different diseases and medical conditions. The importance of these functional medical imaging techniques comes from their ability to monitor abnormal biological behaviors or activities. Photons in the near-IR (NIR) region are modeled as particles which have both absorption and scattering properties when interacting with biological media, such as tissues, cells or organs [1]. Photons suffer broadening and eventual decay as they travel because of multiple scattering and absorption when penetrating and traveling through bio-media; therefore, propagation of these photons within bio-media depends on the optical absorption and scattering properties of the media, which is defined by particles within the cells, cell organelles, and fiber structure [1]. NIR optical parameters of the tissue depend on both absorption and scattering of photons, which reveals information related to oxygenated and de-oxygenated hemoglobin and water concentration [1-2]. The functional near-infrared (fNIR) imaging is used to determine an important aspect of biological media functionality by spectroscopic measurements that are related to the concentration of oxygen and de-oxygenated hemoglobin, blood flow rate, and the amount of water when photons have penetrated and traveled in bio-media. The photons are modeled as particles that are modulated at radio frequency signals and experience absorption and scattering by diffused media. The advantage of using broadband frequency domain is that modulated photons travel in different depths depending on the modulation frequency. Amplitude and phase of both forward and back-scattered photons out of the biological media are measured as IL and IP of the radio frequency signal and then Diffusion Equation is employed for inverse problem solving of turbid biological media. The advantages of broadband measurements 
are already reported [3-5] and are due to high accuracy in extraction of optical parameters and imaging of inhomogeneous material represented as multilayer media.

This paper presents a clinical study of both ganglion cysts and lipoma fatty tumors using the non-invasive fNIR method, where broadband frequency modulation of photons in the NIR region non-invasively diagnoses the condition of these two hand tumors. This method would eliminate the use of an invasive biopsy and would distinguish ganglion cysts from adipose base tumors such as lipomas.

A ganglion cyst is a tumor that is caused by soft tissue swelling, and may occur in any location on the hand and wrist [6-7]. Ganglion cysts begin from the capsule of a joint or a sheath of a tendon, and usually occur between the fibers causing localized enlargement. A lipoma is an adipose fatty tumor that can appear in any part of the human body. It is a fatty lump that grows slow and appears in both soft and hard enlargement under the skin. Accurate diagnosis of these tumors can help define the accurate treatment since the risk of developing a malignant tumor is significant in such medical conditions. Treatment of both ganglion cysts and lipoma tumors would depend on the factor of knowing the physiological properties of the both the cyst and fat [8-9].

This paper investigates both conditions as part of the early diagnosis using broadband functional NIR imaging and reports the relation between reduced scattering coefficient and three photon wavelengths of $670 \mathrm{~nm}, 795 \mathrm{~nm}$ and $850 \mathrm{~nm}$. Performance of non-invasive backscattering photon migration of the tumor is applied to investigate and distinguish between different types of tumors. Extraction of the optical properties of the tumors using forward scattering of the photons is also applied at three wavelengths of $670 \mathrm{~nm}, 795 \mathrm{~nm}$ and $850 \mathrm{~nm}$.

\section{Method}

The overall fNIR imaging system consists of multi wavelength optical source and optical receiver, along with vector network analyzer (VNA) to be used as high dynamic range RF receiver as shown in Figure 1.

Three sources of photons in the wavelength of $670 \mathrm{~nm}, 795 \mathrm{~nm}$, and $850 \mathrm{~nm}$ are used as an optical transmitter. The tri-wavelength photon source is operated by an SP3T switch and separate DC biasing control for each wavelength source. The customized optical Tx used the RF switch from Hittite (HMC245QS16) to drive three high-power vertical cavity surface emitting lasers (VCSELs) $(670 \mathrm{~nm}, 795 \mathrm{~nm}, 850 \mathrm{~nm})$. The tri-wavelength VCSEL commercially available from VIXAR (Module V3WLM) is a high-power laser that has a threshold current of $8.5 \mathrm{~mA}$ for the $670 \mathrm{~nm}, 9.5 \mathrm{~mA}$ for the $795 \mathrm{~nm}$, and $2.8 \mathrm{~mA}$ for the $850 \mathrm{~nm}$. A printed board has been designed and fabricated using a commercial substrate (FR4) as shown in Figure 2, to accommodate all surface mount technology (SMT) components. In the absence of commercial high gain optical receivers using PIN Photodiode, APD, Figure 2, from Hamamatsu (ADP module C5658) has been used along with a built-in trans-impedance amplifier. This module provides high sensitive optical measurements with spectral response between 400-1000nm and the built-in TIA gives $34 \mathrm{~dB}$ of gain up to $1 \mathrm{GHz}$. The overall system is based on a practical free-space system that would eliminate the noise caused by bulky fiber optic systems due to the patient moving during the measurements and discomfort.

This developed VNA based system [10-11] has the ability to monitor modulated photons forward- and back-scattering from the turbid medium using the measured $\mathrm{S}_{21}$ (i.e., IL and IP). Using a proper calibration of the frequency modulated tri-wavelength optical transmitter (VCSEL) and optical receiver (APD C5658 from Hamamatsu), optical tissue parameters are to be extracted using curve fitting the experimental data to $\boldsymbol{a} \sqrt{\text { frequency }}+\boldsymbol{b}$ [5]. The diffusion of NIR photons is impacted by absorption and scattering of the inhomogeneous biological tissue of the wrist with the tumor. 
Human patients diagnosed with both benign ganglion cysts and lipomas are used in this study. Hand and wrist ganglion cysts and lipomas of six patients were imaged using the fNIR system to measure the IL and IP. The study is divided into two stages. The first stage is dedicated to performing functionality measurements to monitor both absorption and scattering, while the second stage is dedicated to optical parameter extraction of the ganglion cyst tissue and lipoma fatty tumor. The first stage is performed while the patients is bending his wrist to measure photon backscattering as shown in Figure 2, while the second stage is performed using transmission mode optical parameter extraction [3-5] after the ganglion cyst fluid and fatty tumor are aspirated.

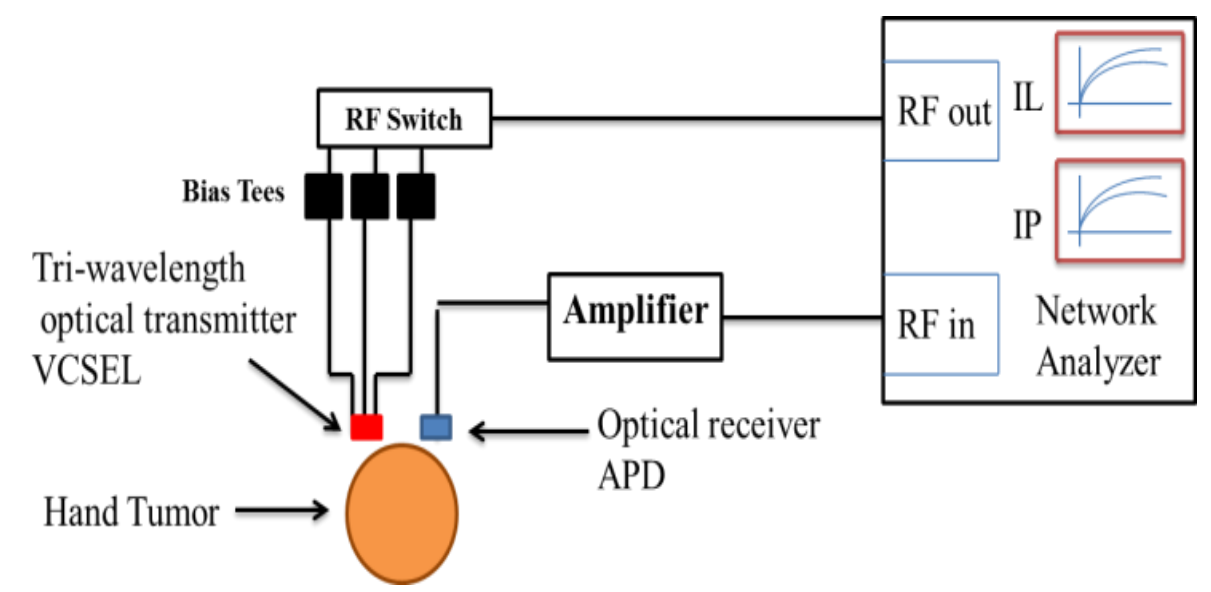

Figure 1. Overall Block Diagram of a Tri-Wavelength fNIR System

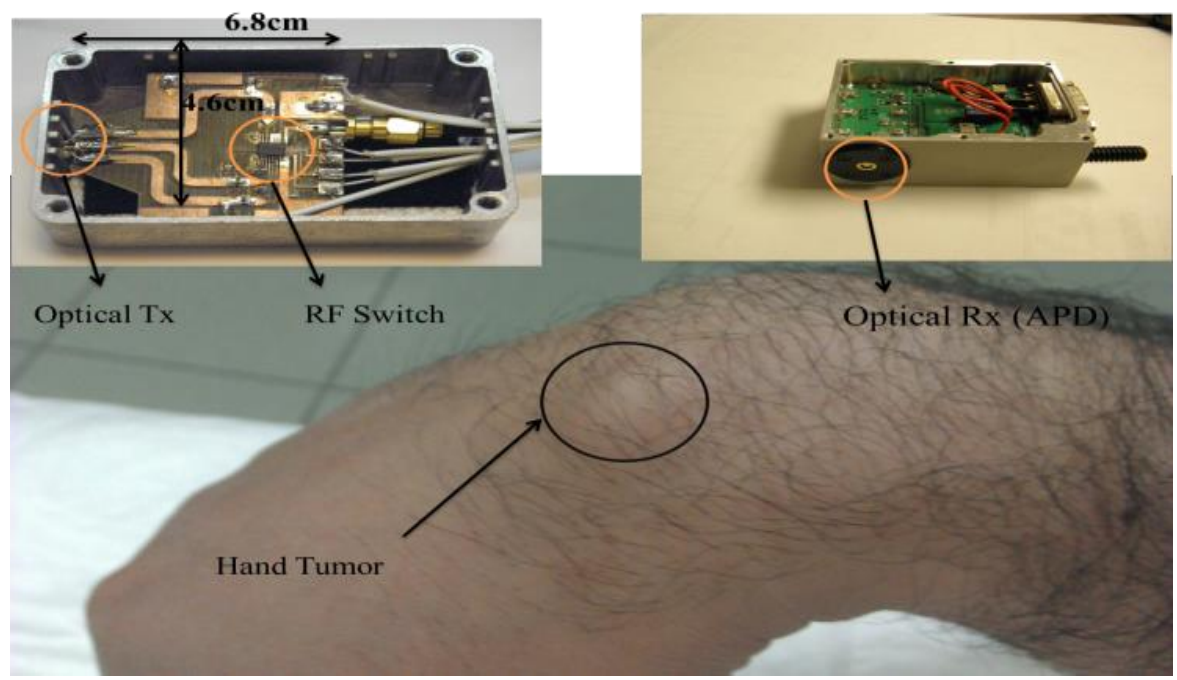

Figure 2. Patients Bend Wrist for Photon Backscattering Measurement

\section{Optical Parameter Extraction using DE and FEM Modeling}

The transmission mode technique is used to extract optical parameters. The transmission mode technique was performed after the ganglion fluid and fatty tumor is surgically extracted for treatment. The experimental data were then used to perform the inverse problem solving using Diffusion Equation (DE) in order to extract the optical parameters of the fluid and fat. The DE of homogeneous media is used to model frequency modulated photon migration in a uniform homogeneous media by extending the Boltzmann transport theory [12]. It has been demonstrated that the DE could predict a behavior as accurate as the Monte Carlo (MC) technique for RF modulation frequencies 
up to $1 \mathrm{GHz}$ [13]. The Diffusion Equation has been used in our analysis for broadband frequency modulation analysis and extraction of the optical parameters. The Diffusion Equation for a homogeneously distributed turbid media is expressed as follows:

$$
\frac{1}{c} \frac{\partial \phi(r, t)}{\partial t}-D \nabla^{2} \phi(r, t)-\left(\mu_{a}\right) \phi(r, t)=S(r, t)
$$

where $\emptyset(r, t)$ is the fluency rate $\left(\mathrm{w} / \mathrm{mm}^{2}\right)$ for reflectance or transmittance mode, and $\mathrm{c}$ is the speed of light in the media. For a sinusoidal point source modulated at angular frequency in a semi-infinite medium, the photon density wave (PDW) is expressed as [1415]:

$$
\begin{gathered}
\emptyset(r, t)=\frac{A_{d c}}{4 \pi D} \frac{\mathrm{e}^{\left(-\frac{r}{\delta}\right)}}{r}+\frac{A_{a c}}{4 \pi D} \frac{\mathrm{e}^{\left(-k_{\text {imag }} r\right)}}{r} e^{\left.\left[-j\left(k_{\text {real }} r-\omega t\right)\right)\right]} \\
K_{\text {real }}=\sqrt{\frac{3}{2} \mu_{a} \mu_{s}^{\prime}}\left\{\left[1+\left(\frac{\omega}{c \mu_{a}}\right)^{2}\right]^{\frac{1}{2}}+1\right\}^{\frac{1}{2}} \\
K_{\text {imag }}=\sqrt{\frac{3}{2} \mu_{a} \mu_{s}^{\prime}}\left\{\left[1+\left(\frac{\omega}{c \mu_{a}}\right)^{2}\right]^{\frac{1}{2}}-1\right\}^{\frac{1}{2}}
\end{gathered}
$$

where $A_{d c}$ and $A_{a c}$ are respectively the DC (unmodulated) and AC (modulated) components of the source, $\delta$ is the light penetration depth for unmodulated light, and $k_{\text {real }}$ and $k_{\text {imag }}$ are the real and imaginary components of complex wave number that describes spatial evolution for the PDW. Parameter $\mathrm{D}$ is the diffusion coefficient $D=\frac{1}{3 \cdot \mu_{s}^{\prime}}$ and $\mathrm{r}$ is the separation distance between transmitter and receiver. The analytical solution was used to compare the experimental measurements and then extract both absorption $\mu_{\mathrm{a}}$ and scattering $\mu_{\mathrm{s}}^{\prime}$ coefficients using the least square error methods (lsqcurvefit) in Matlab. The least square curve fitting method is based on Levenberg-Macquart, which is used to minimize the merit function:

$$
\chi^{2}=\sum_{i}\left(\frac{A_{n, i}-A_{\text {theory }}\left(\mu_{a}, \mu_{s}^{\prime}\right)}{A_{\text {theory }}}\right)^{2}+\left(\frac{P_{n, i}-P_{\text {theory }}\left(\mu_{a}, \mu_{s}^{\prime}\right)}{P_{\text {theory }}}\right)^{2}
$$

$\mathrm{A}$ and $\mathrm{P}$ represent amplitude and phase of the measured data respectively. The FEM model is then used to simulate the behavior of frequency modulated photons at wavelengths of $670 \mathrm{~nm}, 795 \mathrm{~nm}$ and $850 \mathrm{~nm}$, when it is interacted with the ganglion cysts and lipoma fat. Commercial multi-physics based FEM software (COMSOL) is used as a robust numerical tool in this study to numerically predict the photon behavior in scattering media and to solve the inverse problem. The Helmholtz equation is used for numerical analysis [4][16-17] as:

$$
\nabla(-D \nabla \emptyset(r, t))+\mu_{a} \emptyset(r, t)=S(r, t)
$$

where $\emptyset(r, t)$ is the photon flux, $D=\frac{1}{3 \cdot \mu_{s}^{\prime}}, S(r, t)=S_{O}\left(1+m e^{j \omega t}\right)$ is a frequency modulated NIR system where $m$ is amplitude modulation index at time harmonic modulation frequency of $\omega$ The COMSOL representation of the Helmholtz equation is:

$$
-\nabla \cdot(c \nabla u)+a u=f
$$

Diffusion parameters of inhomogeneous layers are listed in Table 1. It has been our experience that having mesh structures of element sizes of $80 \mathrm{~nm}$ and $1000 \mathrm{~nm}$ in regions around optical $\mathrm{Tx} / \mathrm{Rx}$, in open bounderies, and everywhere else respectively, would predict an accurate result (e.g., error of less than 5\%) of amplitude and phase change. A typical computation time of 4 seconds for single 2D simulation and 24-28 second for single 3D simulation is experienced using an Intel Core i3 processor PC. These meshing 
element sizes are also recommended for interfaces, when multi-layer human head phantoms are modeled [4]. Table 2. Show the meshing resolution and processing time used for performing numerical solution in 2D and 3D for both ganglion and lipoma.

Table 1. Coefficients and Relevant Expressions used in Helmholtz SubDomains that Govern each Layer of Skin and Tumor

\begin{tabular}{ccc}
\hline & Coefficient "c" & Coefficient "a" \\
\hline Layer 1 & $\frac{1}{3\left(\mu_{a 1}+\mu_{s 1}^{\prime}\right)}$ & $\mu_{a 1}$ \\
Layer 2 & $\frac{1}{3\left(\mu_{a 2}+\mu_{s 2}^{\prime}\right)}$ & $\mu_{a 2}$ \\
\hline
\end{tabular}

Table 2. 3D Numerical Simulation Processing Time for Meshing Structure of $80 \mathrm{~nm}$ Around Optical Tx/Rx and 1000nm everywhere else at Wavelengths of $670 \mathrm{~nm}, 795 \mathrm{~nm}$ and $850 \mathrm{~nm}$ for both Lipoma and Ganglion Tumors

\begin{tabular}{lll}
\hline Wavelength & Lipoma & Ganglion \\
\hline $\mathbf{6 7 0 n m}$ & 28 seconds & 28 seconds \\
\hline $\mathbf{7 9 5 n m}$ & 26 seconds & 27 seconds \\
\hline $\mathbf{8 5 0 n m}$ & 25 seconds & 24 seconds \\
\hline
\end{tabular}

In COMSOL, the boundary condition is set either through Dirichlet or Neumann boundary conditions, where they are set to express the fluency rate, $u$, at desired boundaries. Dirichlet and Neumann boundary conditions are chosen appropriately for airdielectric (or dielectric-dielectric) and radiation conditions in regions surrounding biomaterials. The mathematical representation is expressed [4] as:

$$
\begin{gathered}
n \cdot\left((c \nabla u)_{1}-\left((c \nabla u)_{2}\right)+q \cdot u=g\right. \\
h \cdot u=r
\end{gathered}
$$

where $h, r, q$ and $g$ are the boundary coefficients for the phantom modeling. For the diffused photon flux, $h=1$ and $r=0$. For the matching boundary of air-dielectric and dielectric-dielectric interfaces, $\mathrm{q}=0$ and $\mathrm{g}=0$. It is important to mention that extrapolated boundary conditions take into account the biomaterial-air boundary as a radiation boundary by setting the fluency rate to zero using Eq. 9 without any impact on error achieved [4].

The 3D COMSOL result of IL is obtained using the extracted result and depicted in Figure 3. The tumor size considered for this simulation is $2.5 \mathrm{~cm}$ in diameter, which was the real case scenario for all the patients we used in our study.

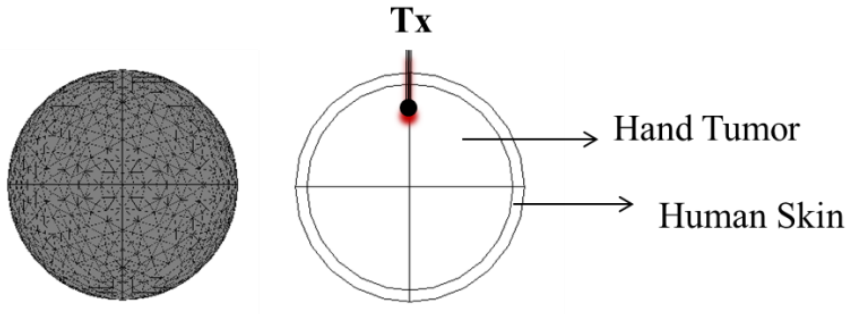

Figure 3. Spherical Shape that Resembles Human Hand Tumor with Meshing Structure 


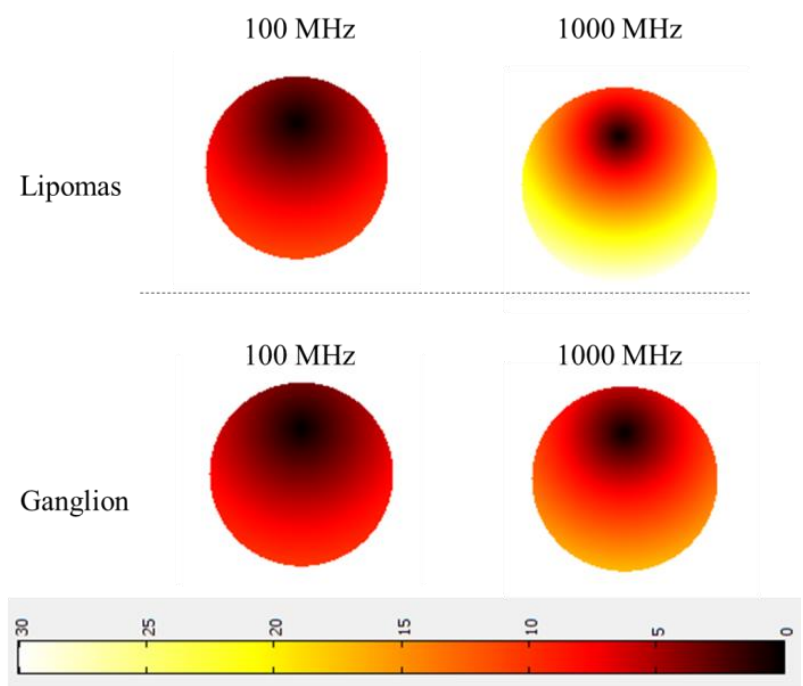

Figure 4. 3D Simulated Broadband Photon Density of IL at $100 \mathrm{MHz}$ and $1000 \mathrm{MHz}$ using FEM Modeling of both Ganglion Cysts and Lipomas at $795 \mathrm{~nm}$, Legend is Showing Scale of the Simulation that Start from 0 to $30 \mathrm{~dB}$

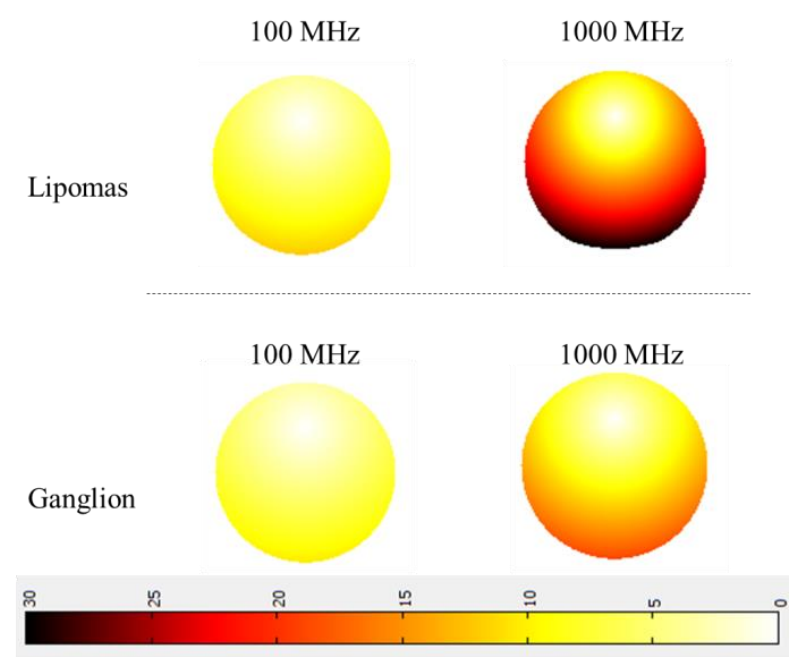

Figure 5. 3D Simulated Broadband Photon Density of IP at $100 \mathrm{MHz}$ and $1000 \mathrm{MHz}$ using FEM Modeling of both Ganglion Cysts and Lipomas at $795 \mathrm{~nm}$, Legend is Showing Scale of the Simulation that Start from 0 to $30 \mathrm{~dB}$

\section{Result and parameter extraction}

Measurements and FEM based simulation of IL and IP of six patients with ganglion cysts and six patients with lipomas are depicted in Figures 4-6. As mentioned in the previous section, the measurements were divided into two stages. The first stage dealt with measuring IL and IP for the reflection mode of operation, while the second stage dealt with optical parameter extraction using transmission mode operation. The procedure was as follows:

Stage 1: The patient bent his wrist so the ganglion cyst or lipoma tumor was obviously seen. The optical transmitter and receiver was then placed $1 \mathrm{~cm}$ apart across the tumor, and broadband frequency modulation measurements were performed, and both IL and IP 
collected for all six patients. The result was then compared to the 3D FE model result performed by COMSOL.

Stage 2: After performing the surgical treatment and aspirating the ganglion cyst fluid and lipoma fatty tumor, the fNIR transmission mode measurements were performed. The purpose of this stage is to extract the optical properties of the fluid and fat. Table 3 shows the result of extracted optical parameters for six patients at three wavelengths of $670 \mathrm{~nm}$, $795 \mathrm{~nm}$, and $850 \mathrm{~nm}$. Extracting the optical parameters is done by curve fitting the experimental broadband result of both IL and IP data to $\boldsymbol{a} \sqrt{\text { frequency }}+\boldsymbol{b}$. The curve fitting result was then used through the inverse problem code to extract the optical parameters [5].

The method of optical imaging using broadband fNIR was used on a total of 12 patients who were diagnosed with either ganglion cysts or lipomas. Pathological diagnoses are also performed after the surgical treatment and removal of cyst fluid or fatty tissue. Comparison between pathological diagnoses and optical properties of different tumor (cyst or fat) is shown in Table 4. This paper focuses on the reduced scattering coefficient $\left(\mu_{s}^{\prime}\right)$ because both ganglion cyst and lipoma tumor are considered benign and therefore high blood absorption is not present in these medical conditions.
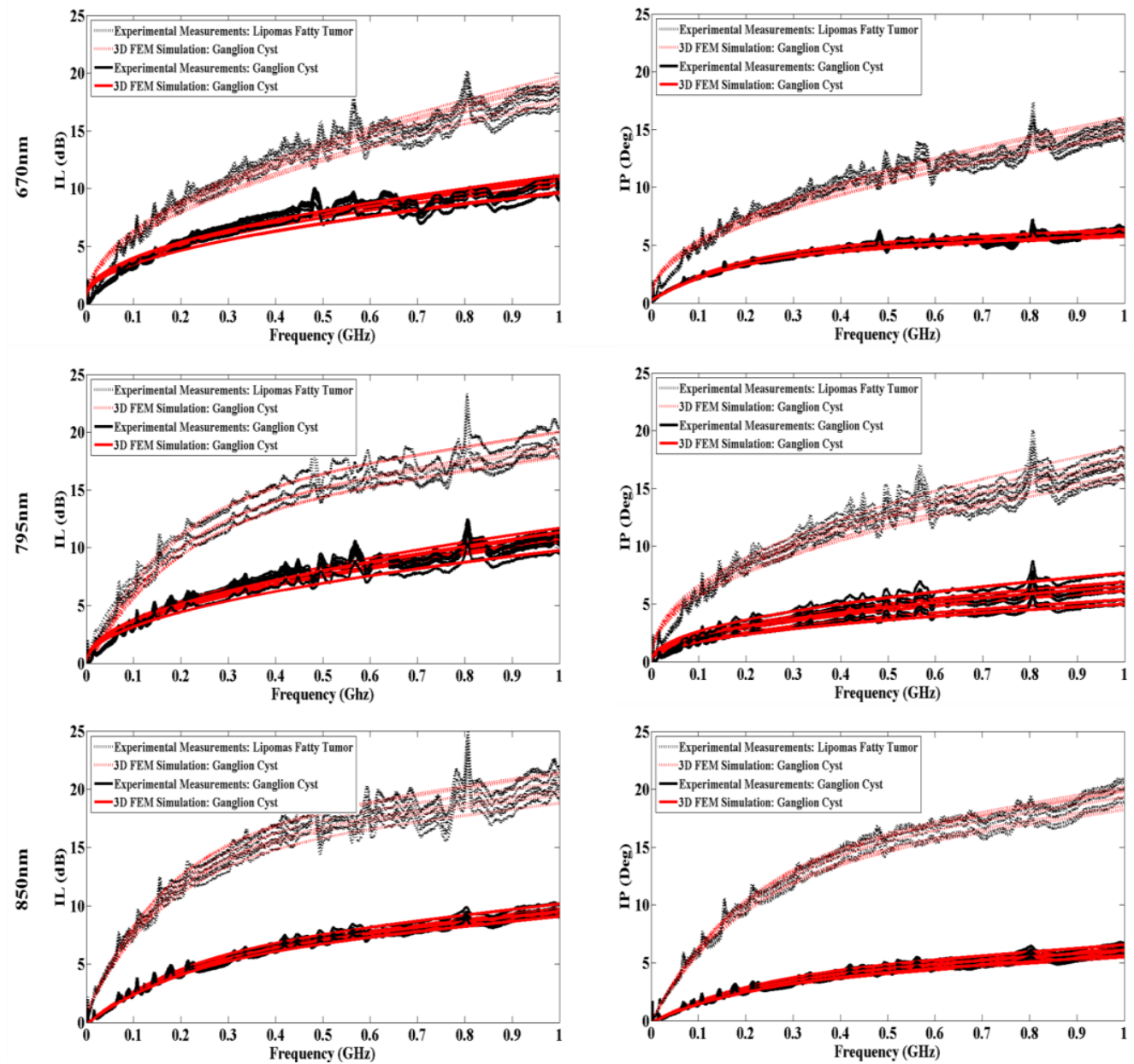

Figure 6. Measured and Simulated IL and IP for Six Patients with Hand Ganglion Cyst and Lipomas at Three Wavelengths of $670 \mathrm{~nm}, 795 \mathrm{~nm}$, and $850 \mathrm{~nm}$ 
Table 3. Extracted Optical Parameters of Ganglion Cyst Fluid using Transmission Mode Broadband Measurements at $670 \mathrm{~nm}, 795 \mathrm{~nm}$ and $850 \mathrm{~nm}$

\begin{tabular}{|c|c|c|c|c|c|c|c|c|c|c|c|c|}
\hline \multirow{3}{*}{ Patients } & \multicolumn{4}{|c|}{$670 \mathrm{~nm}$} & \multicolumn{4}{|c|}{$795 \mathrm{~nm}$} & \multicolumn{4}{|c|}{$850 \mathrm{~nm}$} \\
\hline & \multicolumn{2}{|c|}{ Ganglion Cysts } & \multicolumn{2}{|c|}{ Lipomas } & \multicolumn{2}{|c|}{ Ganglion Cysts } & \multicolumn{2}{|c|}{ Lipomas } & \multicolumn{2}{|c|}{ Ganglion Cyst } & \multicolumn{2}{|c|}{ Lipomas } \\
\hline & $\begin{array}{l}\mu_{a} \\
\left(\frac{1}{c m}\right)\end{array}$ & $\begin{array}{l}\mu_{s}^{\prime} \\
\left(\frac{1}{c m}\right)\end{array}$ & $\begin{array}{c}\mu_{a} \\
\left(\frac{1}{c m}\right)\end{array}$ & $\begin{array}{c}\mu_{s}^{\prime} \\
\left(\frac{1}{c m}\right)\end{array}$ & $\begin{array}{c}\mu_{a} \\
\left(\frac{1}{c m}\right)\end{array}$ & $\begin{array}{c}\mu_{s}^{\prime} \\
\left(\frac{1}{c m}\right)\end{array}$ & $\begin{array}{c}\mu_{a} \\
\left(\frac{1}{c m}\right)\end{array}$ & $\begin{array}{c}\mu_{s}^{\prime} \\
\left(\frac{1}{c m}\right)\end{array}$ & $\begin{array}{c}\mu_{a} \\
\left(\frac{1}{c m}\right)\end{array}$ & $\begin{array}{c}\mu_{s}^{\prime} \\
\left(\frac{1}{c m}\right)\end{array}$ & $\begin{array}{c}\mu_{a} \\
\left(\frac{1}{c m}\right)\end{array}$ & $\begin{array}{c}\mu_{s}^{\prime} \\
\left(\frac{1}{c m}\right)\end{array}$ \\
\hline 1 & 0.085 & 1.2 & 0.71 & 7.8 & 0.074 & 1.4 & 0.78 & 9.2 & 0.083 & 1.42 & 0.83 & 11.2 \\
\hline 2 & 0.075 & 1.61 & 0.74 & 7.9 & 0.068 & 1.23 & 0.81 & 9.6 & 0.072 & 1.56 & 0.81 & 10.8 \\
\hline 3 & 0.072 & 1.72 & 0.73 & 8.3 & 0.082 & 1.5 & 0.86 & 10 & 0.068 & 1.49 & 0.75 & 10.5 \\
\hline 4 & 0.082 & 1.2 & 0.68 & 8.0 & 0.081 & 1.73 & 0.75 & 9.3 & 0.089 & 1.8 & 0.64 & 11.1 \\
\hline 5 & 0.086 & 1.61 & 0.82 & 7.9 & 0.078 & 1.54 & 0.9 & 10.2 & 0.09 & 1.67 & 0.67 & 10.9 \\
\hline 6 & 0.071 & 1.81 & 0.69 & 8.2 & 0.074 & 1.63 & 0.72 & 9.9 & 0.081 & 1.83 & 0.65 & 11.5 \\
\hline
\end{tabular}

\section{Discussions \& Conclusion}

The results presented in the previous section depict that absorption coefficient and that reduced scattering coefficient of both ganglion cyst fluid and lipoma fatty tumor varies significantly between fluid-based and fat-based tumor. Ganglion cysts have a minimum absorption coefficient of $0.068 / \mathrm{cm}$ and a maximum of $0.09 / \mathrm{cm}$, while it has a minimum reduced scattering coefficient of $1.2 / \mathrm{cm}$ and maximum of $1.83 / \mathrm{cm}$. The fat-based tumor (lipoma) has a maximum absorption coefficient of $0.64 / \mathrm{cm}$ and maximum of $0.9 / \mathrm{cm}$, while it has a minimum reduced scattering coefficient of $7.8 / \mathrm{cm}$ and maximum of $11.5 / \mathrm{cm}$. All 12 patients were examined using the back-scattering method of optical imaging using fNIR and results are shown in Figure 6. Here it is clear that patients with lipoma fatty tumors have high IL and IP over the broadband measurements of $30 \mathrm{MHz}$ to $1000 \mathrm{MHz}$, and these results are confirmed when the forward measurements and optical parameters extraction were performed. The numerical 3D FEM simulations were performed based on the optical parameters extracted for all patients and are shown in Figure 6. The simulation gives confidence in the 3D FEM modeling and can be used as a tool for future prediction and diagnoses. Since these benign tumors have low blood absorption, it is recommended to monitor the Insertion Phase of the back-scattering measurements, and so Table 3 shows a comparison between the reduced scattering coefficients which is related to IP and the pathological diagnoses reported after the tumors are extracted. From Table 4 we can identify the type of tumor based on the amount of scattering photons, as the photon is more scattered when it's migrating through hard biomaterials. The soft bio-materials such as ganglion fluid will result in low reduced scattering coefficients while hard bio-materials such as fat will result in high reduced scattering coefficients. The ganglion fluid has very close NIR properties to the transparent fluids in the body such as cerebrospinal fluid (CSF) while the fat tissue has close NIR properties to human bone in the body. Diagnosing ganglion cysts fluid and lipoma fat tumors without invasive biopsy requires knowledge of other types of cysts, tumors and bone optical properties. As Table 4 shows, the reduced scattering coefficient of the ganglion cyst ranges from 1.2 to $1.831 / \mathrm{cm}$ while the reduced scattering coefficient of the lipomas ranges from 7.8 to $11.51 / \mathrm{cm}$, and from the back-scattering measurements of the IP a distinguishable tumor can be identified. 


\section{Table 4. Pathological Diagnoses and Reduced Scattering Coefficients}

\begin{tabular}{|c|c|c|}
\hline Patients & $\begin{array}{c}\text { Extracted optical } \\
\text { parameters at } 670 \mathrm{~nm} \text {, } \\
795 \text {, and 850nm } \\
\mu_{s}^{\prime}\left(\frac{1}{\mathrm{~cm}}\right)\end{array}$ & $\begin{array}{c}\text { Pathological } \\
\text { Diagnoses }\end{array}$ \\
\hline 1 & $1.2-1.42$ & Ganglion Cyst \\
\hline 2 & $1.23-1.61$ & Ganglion Cyst \\
\hline 3 & $1.49-1.72$ & Ganglion Cyst \\
\hline 4 & $1.2-1.8$ & Ganglion Cyst \\
\hline 5 & $1.54-1.67$ & Ganglion Cyst \\
\hline 6 & $1.63-1.83$ & Ganglion Cyst \\
\hline 7 & $7.8-11.2$ & Lipoma \\
\hline 8 & $7.9-10.8$ & Lipoma \\
\hline 9 & $8.3-10.5$ & Lipoma \\
\hline 10 & $8.0-11.1$ & Lipoma \\
\hline 11 & $7.9-10.9$ & Lipoma \\
\hline 12 & $8.2-11.5$ & Lipoma \\
\hline
\end{tabular}

\section{Acknowledgment}

This work was partially supported by the PAAET research grant number (TS10-009), and research grant number (TS14-09). The authors (ES and NA) would like to thank Kuwait University Electrical Engineering Department RF lab, Al Razi Orthopedic Hospital, and the Public Authority for Applied Education and Training for their support.

\section{References}

[1] C. Mansouri, "New Window on Optical Brain Imaging; Medical Development, Simulations and Applications", Selected Topics on Optical Fiber Technology, (2012).

[2] B. L. Edlow and O. Wu, "Advanced Neuroimaging in Traumatic Brain Injury", SeminNeurol, vol. 32, no. 04, (2012), pp. 374-400.

[3] E. Sultan, "Accurate optical parameter extraction procedure for broadband near-infrared spectroscopy of brain matter", Journal of Biomedical Optics, vol. 18, no. 1, (2013), pp. 017008-017008.

[4] E. Sultan, "3D Numerical modeling and its experimental verifications for an inhomogeneous head phantom using broadband fNIR system", International Journal for Numerical Methods in Biomedical Engineering, (2013).

[5] E. Sultan, K. Manseta, A. Khwaja, L. Najafizadeh, A. Gandjbakhche, K. Pourrezaei and A. S. Daryoush, "Modeling and tissue parameter extraction challenges for free space broadband fNIR brain imaging systems", Proceeding of SPIE 7902, 790223, (2011).

[6] W. E. Barnes, "Review of ganglia of the hand and wrist with analysis of surgical treatment", Plastic and Reconstructive Surgery, vol. 34, no. 6, (1964), pp. 570-578.

[7] M. Suen, "Treatment of Ganglion Cysts", ISRN Orthopedics 2013, (2013).

[8] T. T. Miller, H. G. Potter and R. R. LcCormack, "Benign soft tissue masses of the wrist and hand: MRI appearances", Skeletal Radiol, vol. 23, (1994), pp. 327-332.

[9] T. Mcpherson, "Benign tumors of fibrous tissue and adipose tissue of the hand", Journal of Hand Therapy, vol. 18 , no. 1 , pp. 53-54.

[10] M. Khushali, "Development Challenges of Brain Functional Monitoring using Untethered Broadband Frequency Modulated fNIR System", Proceeding of IEEE Microwave Photonics, MWP 2010, Montreal, Canada, pp. 295-299.

[11] E. Sultan, "Tethered and Untethered Helmet Mounted fNIR Systems for TBI Application", 12th annual IEEE Wireless and Microwave Technology (WAMI) Conference, Orlando, USA, (2011) April.

[12] M. S. Patterson, S. J. Madsen and J. D. Moulton, "Diffusion equation representation of photon migration in tissue", IEEE 1991 Microwave Symposium Digest, (1991), pp. 905-908. 
[13] J. B. Fishkin, S. Fantini, M. J. vandeVen and E. Gratton, "Gigahertz photon density waves in a turbid medium: theory and experiments", Phys. Rev. E Stat. Phys. Plasmas Fluids Relat. Interdiscip. Topics, vol. 53, (1996), pp. 2307-2319.

[14] M. S. Patterson, B. Change and B. C. Wilson, "Time resolved reflectance and transmittance for the noninvasive measurement of tissue optical properties", Appl. Opt., vol. 28, no. 12, (1989), pp. 2331-2336.

[15] D. Contini, F. Martelli and G. Zaccanti, "Photon migration through a turbid slab described by a model based on diffusion approximation I. Theory”, Appl. Opt., vol. 36, (1997), pp. 4587-4599.

[16] M. Schweiger, S. R. Arridge, M. Hiraoka and D. T. Delpy, "The finite element method for the propagation of light in scattering media: boundary and source conditions", Med. Phys., vol. 22, (1995), pp. 1779-1792.

[17] S. R. Arridge, M. Schweiger, M. Hiraoka and D. T. Delpy, "A finite element approach for modeling photon transport in tissue", Med. Phys., vol. 20, (1993), pp. 299. 